\title{
OPTIMAL MAKE-UP TORQUE FOR TRAPEZOIDAL THREADED CONNECTIONS SUBJECTED TO COMBINED AXIAL TENSION AND INTERNAL PRESSURE LOADING
}

\author{
Timothy Galle \\ Ghent University \\ Laboratory Soete \\ Technologiepark 903 \\ 9052 Zwijnaarde, Belgium \\ Timothy.Galle@UGent.be \\ Jeroen Van Wittenberghe \\ Arcelor Mittal R\&D Ghent \\ OCAS NV \\ Technologiepark 935 \\ 9052 Zwijnaarde, Belgium \\ Jeroen.VanWittenberghe@arcelormittal.be
}

\author{
Wim De Waele \\ Ghent University \\ Laboratory Soete \\ Technologiepark 903 \\ 9052 Zwijnaarde, Belgium \\ Wim.DeWaele@UGent.be \\ Patrick De Baets \\ Ghent University \\ Laboratory Soete \\ Technologiepark 903 \\ 9052 Zwijnaarde, Belgium \\ Patrick.DeBaets@UGent.be
}

\begin{abstract}
When assembling tubing strings in the oilfields, threaded connections are used to connect the pipes with each other. The possibility to reuse these connections is often required and a certain degree of leak tightness is required, even without the use of a sealing surface or shoulder. For this reason, the total plasticity within the connection should be limited and relative movement between pin and box ought to be restricted.

Within this publication, a finite elements analysis is conducted using a 4.5 inch buttress threaded connection as defined in API 5B together with a connection using the enhanced SR23 buttress thread. In addition, an experimental validation of the make-up stage is conducted by comparing the strains generated during make-up using Digital Image Correlation and infrared monitoring.

In order to determine the optimal make-up, values found in literature are compared with a developed method using the magnitude and size of the plastically deformed zones during make-up. In addition, the effect of external axial and pressure loading is examined to identify the effects and critical areas.

As a result, it is observed that pressure loading and make-up tend to have similar effects and in order to determine the optimal make-up torque, the pressure ratings of the assembly should be taken into account to prevent overtorqueing the connection. For the case of axial loading, a critical zone is visible near the last engaged thread and excessive loading of this thread can cause premature failure within this zone. Overall, the SR23 connection
\end{abstract}

shows limited, yet visible, advantages over the standard BTC connection as described in literature.

$\begin{array}{cll}\text { NOMENCLATURE } & \\ \sigma & \text { Yield strength } & {[\mathrm{MPa}]} \\ A_{p} & \text { Section area } & {\left[\mathrm{mm}^{2}\right]} \\ D & \text { Outer diameter } & {[\mathrm{mm}]} \\ F_{a} & \text { Axial load } & {[\mathrm{N}]} \\ F_{a, \max } & \text { Maximum axial load } & {[\mathrm{N}]} \\ k_{i} & \text { Geometric constant } & {[-]} \\ n_{\text {exp }} & \text { Experimental make-up turns } & {[\text { turns }]} \\ n_{\text {FEA }} & \text { Numerical make-up turns } & {[\text { turns }]} \\ p_{i} & \text { Internal pressure } & {[\mathrm{MPa}]} \\ p_{i, \max } & \text { Maximum internal pressure } & {[\mathrm{MPa}]} \\ t & \text { Wall thickness } & {[\mathrm{mm}]}\end{array}$

\section{INTRODUCTION}

To reduce running times on-site and with the intention of creating leak tight connections, threaded couplings are used when drilling and completing oil and gas wells. One of the available configurations is the Threaded and Coupled (T\&C) geometry which exists of two male pins (usually threaded on the pipes that need to be connected) and an additional female box. Despite the fact that these types of connections have been proven to be reliable and reusable, they still inhibit a major disadvantage. In order to obtain a $100 \%$ performance ratio compared to the pipe body performance, the outer diameter of 
these connections exceeds the outer diameter of the connecting pipes. This increase in dimensions can cause the string to get stuck down-hole when running. In an effort to reduce the outer diameter of the connection, the overall design, thread type and initial preloads (make-up) are being investigated.

This paper discusses a numerical study consisting of a comparison between a standard buttress and an enhanced SR23 connection which is assumed to have a better leak resistance along its thread helix when pressure and axial loads are applied. During this study, a method to determine the minimum and maximum make-up position is suggested with the intention of maximizing the performance of the connection. In addition, leak tightness is assessed using the gap size which is present near the thread flanks.

Despite the commonly accepted approach of using axi-symmetric models to simulate the behavior of threaded connection, the make-up stage is not yet fully validated in literature. Therefore, an experimental setup is designed to validate the make-up procedure. At first, the numerically predicted strains are validated by using digital image correlation and strain gauges. Secondly, the temperature at the outer surface of the assembly is monitored by means of thermography. By assuming that the temperature increase originates from frictional energy, an indication for the distribution of contact pressure can be extracted from the results.

\section{FINITE ELEMENT MODEL}

The threaded contact area is of main importance when trying to describe the leak tightness of a threaded connection, especially the gap sizes between the different flanks. Since it is nearly impossible to monitor this contact interface during experiments, the use of numerical techniques has proven to provide valuable information. A parametric finite element model using ABAQUSTM is developed as part of an extensive study trying to characterize and optimize the geometry of threaded connections. The analysis is based on the use of a simplified 2D axisymmetric model [1] of a threaded connection using the penalty method to incorporate the non-linear contact definitions. The material used for both pin and box was a qualified N80 steel [2] with a yield strength of $730 \mathrm{MPa}$. Further details of the applied modelling approach can be found in [3].

Using this model, twenty equally distributed make-up positions between hand tight and two make-up turns are simulated by means of resolving an adequate amount of initial overlap. In addition to the make-up, external axial tensile and internal pressure loads are applied. Four different loading scenarios are simulated for every make-up position: uniaxial tension, internal pressure and two cases of combined internal pressure and axial tension (see further for the calculations of the $100 \%$ test loads for the cases with combined loading). For every load scenario, a gradual increase of the load by $10 \%$ was applied and output data was extracted at every increment. An overview of the performed simulations for a single make-up position is provided in Figure 1. The use of the modeling method when applying external loads is already widely validated by means of experiments [4,5]. For the make-up state however, very limited information with conclusive prove is available in literature.

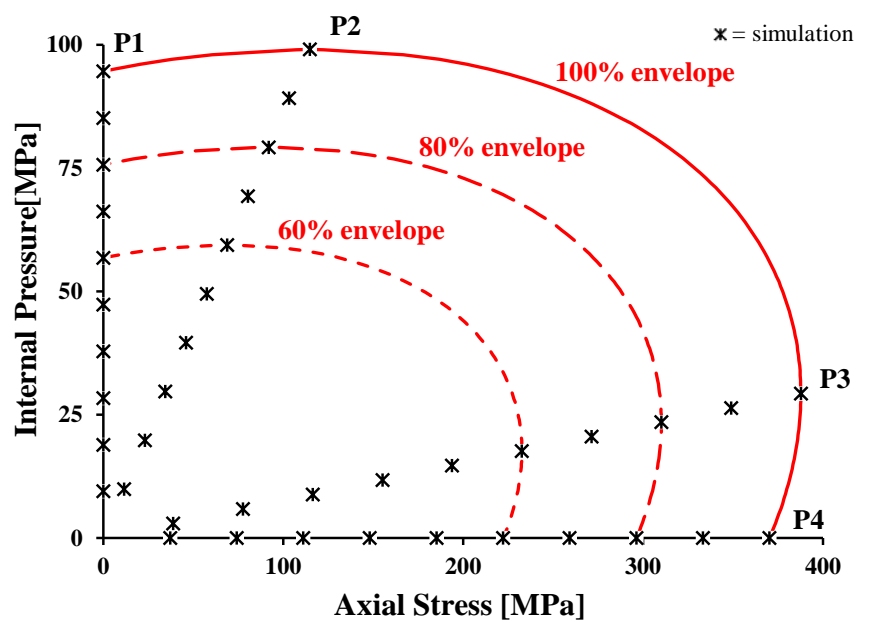

Figure 1: Overview of the Von Mises Ellipse (VME) with every simulation performed per make-up level

Apart from the standard generated output such as stresses, strains and contact pressure, an estimation for the minimum and maximum allowable make-up position together with corresponding make-up torque is provided. To determine the minimal make-up position required to create a reliable and leak tight connection, two parameters are considered: gap size between the thread flanks and the frictional holding torque. To establish a maximum allowable torque position, global plasticity is avoided by monitoring the plastic energy of the connection in function of external load and make-up.

\section{EXPERIMENTAL VALIDATION OF MAKE-UP}

\section{Setup}

Because of the absence of a conclusive validation of using axisymmetric models for simulating make-up conditions in threaded connections, an experimental setup is designed for validation purposes. The setup, given in Figure 2, was designed keeping two different objectives in mind.

The primary objective is to validate the stress-strain state of the made-up connection by measuring the strains at the outer surface of the box using two different measurement techniques. In addition to conventional used strain gauges (type FCA-3-11), an optical technique named digital image correlation (DIC). When using this technique, a random speckle pattern is applied on a free surface of the box (see the section view in Figure 2) which is monitored by two synchronized cameras. By using two cameras, it is possible to obtain a 3D correlation using stereoscopy principles. During the make-up stage, the relative position of these speckles is monitored and analyzed using dedicated software to extract the strains. A more detailed description can be found in [6]. The twelve biaxial strain gauges were attached in three different sections with the intention of 


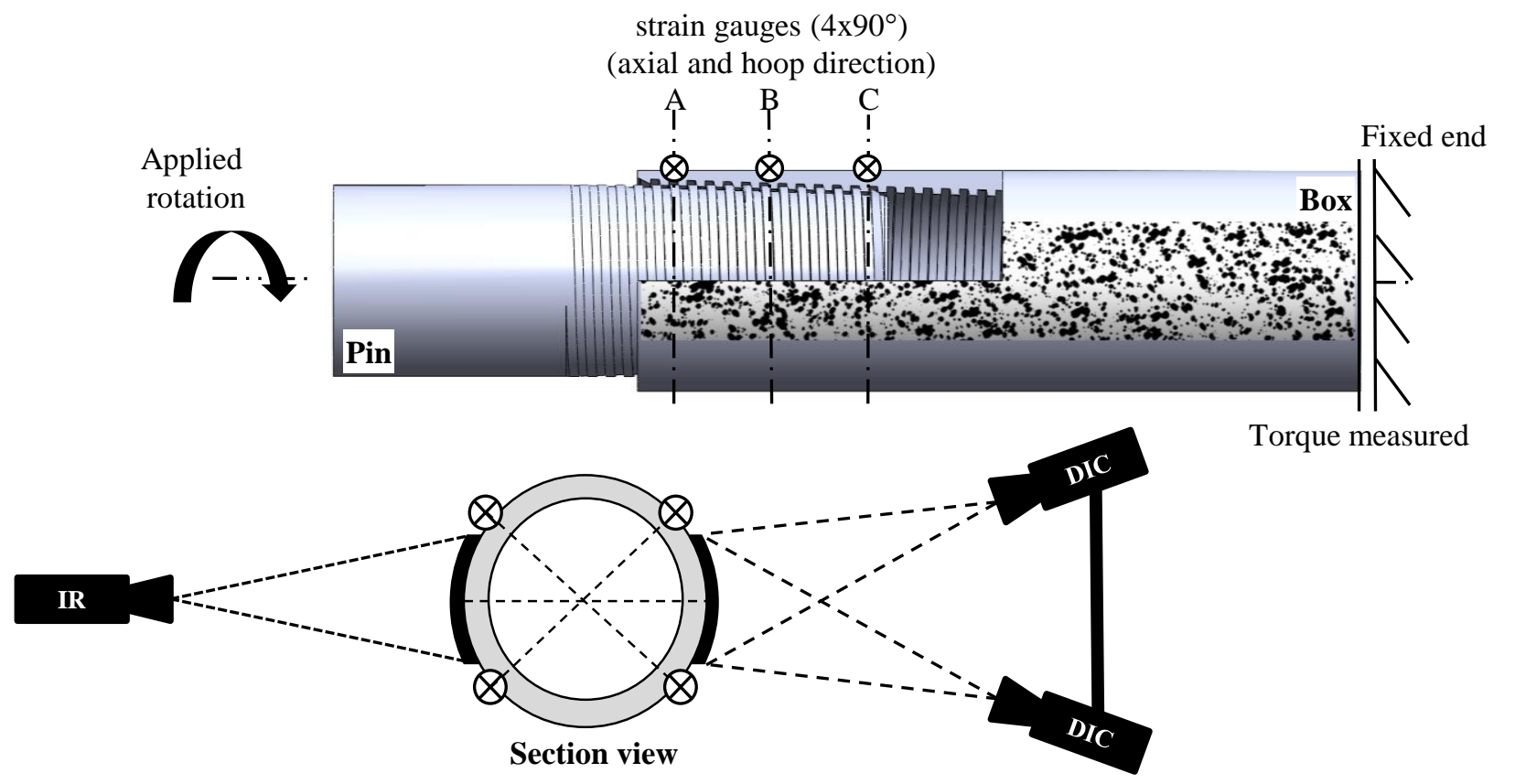

Figure 2: Schematic representation of the experimental setup - Strain gauges are distributed over 3 sections of the box while digital image correlation is used on one side and thermography is used on the other.

validating the values obtained by the DIC approach. Both techniques showed similar results, but the main advantage of using DIC is that a larger area can be investigated at once. Therefore, despite the better accuracy of the strain gauges, it is the preferred method for numerical validation purposes.

A secondary purpose of the setup is to provide information about the contact surface in an effort to validate the predicted contact pressures at the flanks. An initial evaluation of the global contact area can be provided by measuring the torque-turn diagram in which the applied make-up torque is drawn in function of the rotation of the pin and compared with the numerical predicted frictional torque. In addition, the temperature at the surface of the box can be monitored using an infrared (IR) camera (type Infratec 8340). In the assumption that the heat generated during make-up is mainly caused by the frictional energy, the location of the highest occurring contact pressures can be pinpointed. In order to use this technique, a section of the outer surface of the box, opposite to the DIC speckle pattern, is painted with a black emissive spray to increase emission and reduce reflection. This way, a constant emissivity value of 0.93 can be obtained. The thermo graphic camera measures in the mid-wave infrared band $(2.5 \mu \mathrm{m})$ and the thermal resolution NETD is smaller than $25 \mathrm{mK}$. A detailed description of this technique can be found in [8].

\section{Experimental results}

An experiment was conducted using a custom made 2 inch connection containing a tapered API buttress thread. Both pin and box consist of a gradeB material with a yield strength of respectively $370 \mathrm{MPa}$ and $300 \mathrm{MPa}$. A horizontal make-up was applied using the API Modified thread compound on both pin and box and a make-up speed of $0.1 \mathrm{rpm}$ was maintained. During assembly, a picture was taken by the DIC system and IR camera

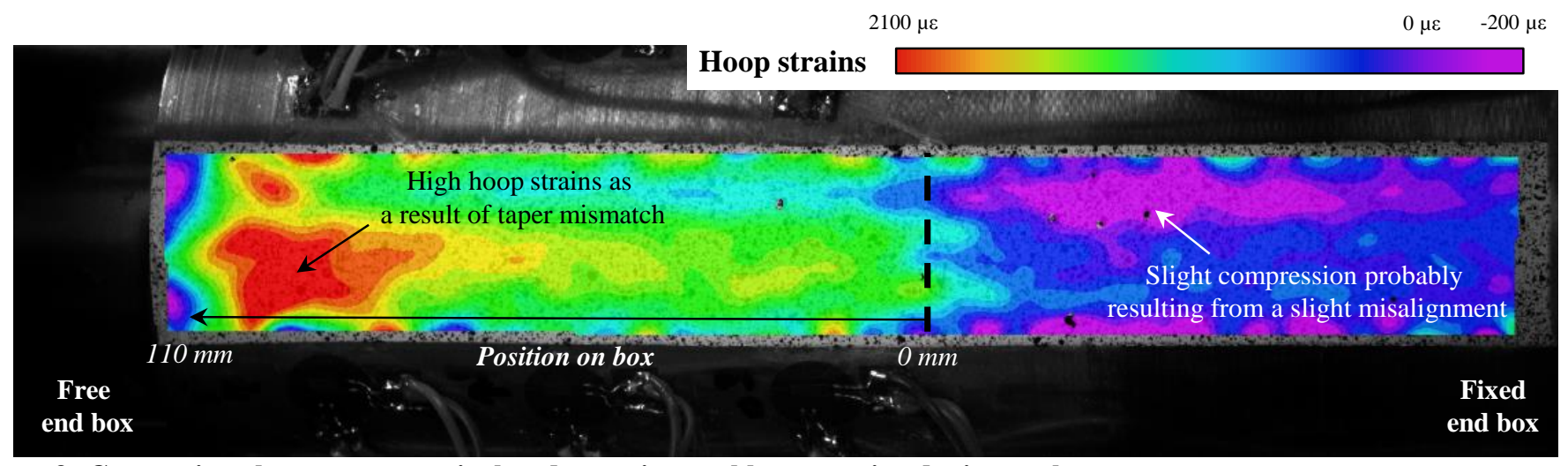

Figure 3: Comparison between numerical and experimental hoop strains during make-up. 
simultaneously every two rotational degrees. Both hoop and axial strains were monitored. An example of the obtained measurements is given in Figure 3. The results of a total of 19 paths parallel with the box axis are extracted and averaged to remove singularities, effects of misalignment and to provide more reliable results. Since axial strains are visible but limited, only the hoop strains are presented in Figure 4 for various makeup levels. From this figure, it is visible that the hoop strains away from the box tip appear to be overestimated for all make-up levels. This behavior is the result of an initial taper mismatch between pin and box. Because the taper of the pin was slightly larger than the taper near the end of the box, the threads at this

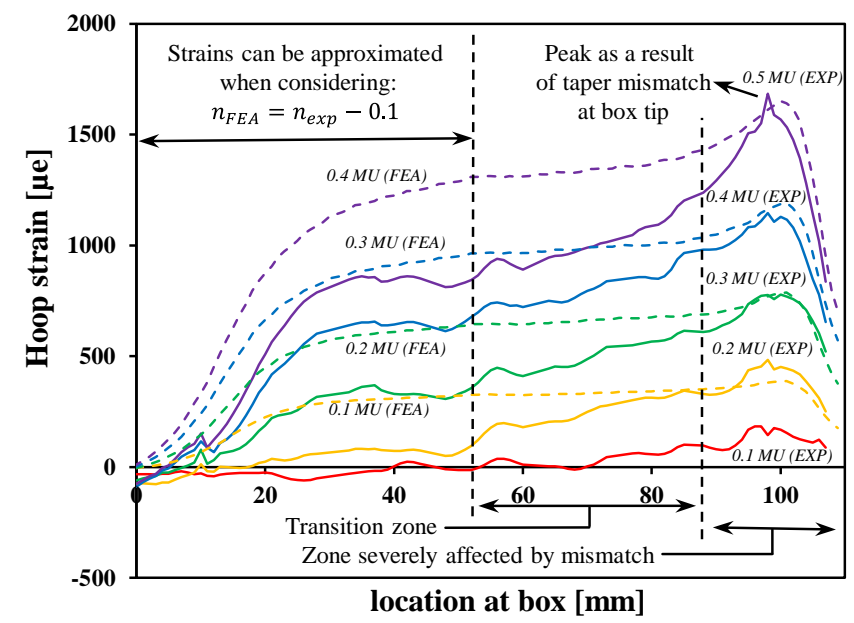

Figure 4: Comparison between numerical and experimental hoop strains during make-up. location made sooner contact, resulting in a slightly higher make-up in comparison with the other part of the thread. From Figure 4 it is visible that the make-up difference is about 0.1 turn.

An example of the thermographic results can be found in Figure 5. Figure 5.A through Figure 5.D show the increase of temperature during make-up. After averaging the temperature over a large number of paths parallel to the box axis at the outer surface of the box, a typical temperature distribution is obtained and represented in Figure 5.E after 0.3 turns make-up. From this figure, it can be observed that the location of the maximum temperature coincides with the location of the maximum average contact pressure in the contact area. The assumption that the temperature increase is mainly caused by frictional heat and not by deformation energy [7] can be backed up by the fact that the location of maximum strains (see Figure 4) and the maximum temperature are not situated at the same position at the box.

\section{NUMERICAL RESULTS}

With the intention of determining the minimum and maximum make-up position in which the connection can reach its $100 \%$ performance rating, different numerical simulations were conducted as mentioned before. Two different geometries are modelled. At one hand the original standard buttress connection and at the other hand an improved version which is used when enhanced leak resistance is required. The details of the thread used in the SR23 connection is given in Figure 6. When compared to the standard buttress thread form [9], the maximum allowable gap size between the matching stab flanks in the hand tight position is reduced by $23 \mu \mathrm{m}$ down to $127 \mu \mathrm{m}$ instead of the previously allowed $150 \mu \mathrm{m}$, when taking into account the geometric tolerances.
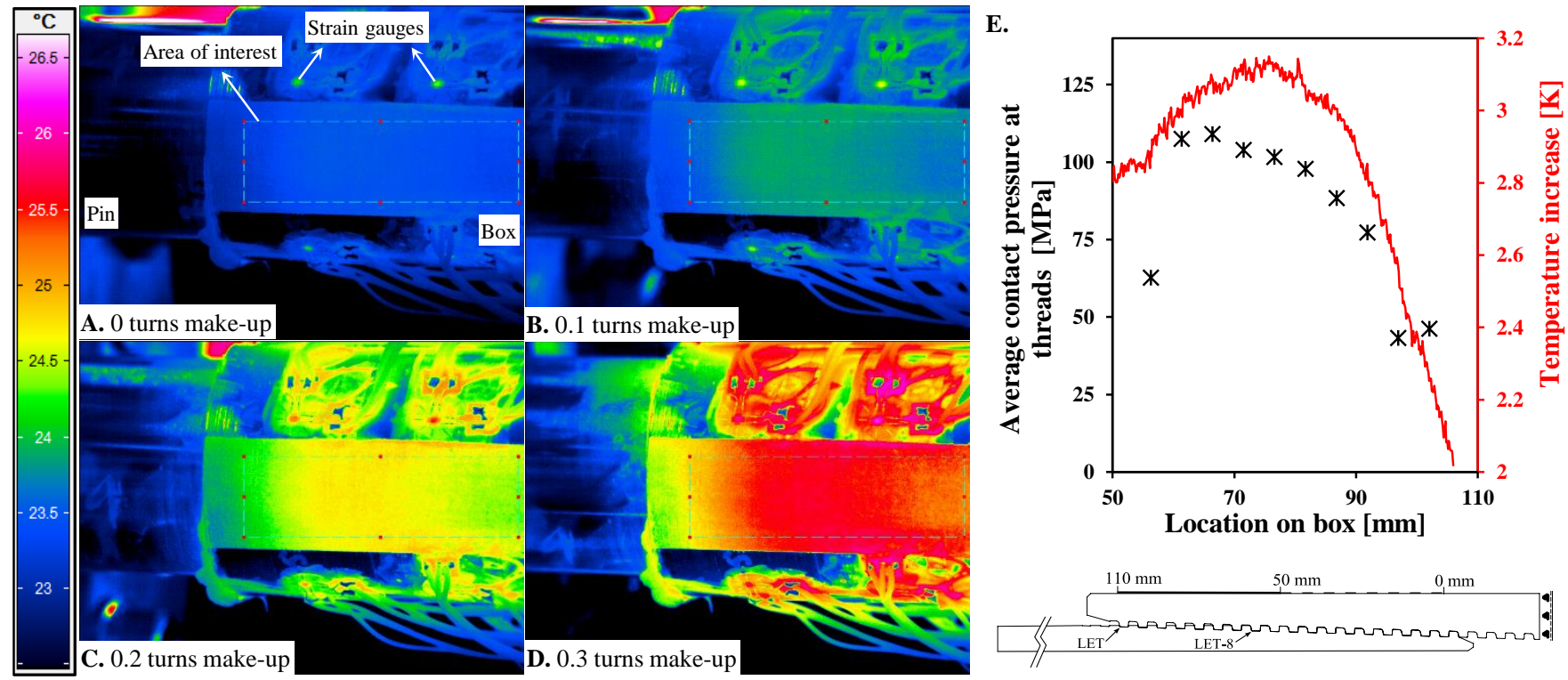

Figure 5: Evolution of temperature in the connection when made up, measured by the infrared camera (A through D). Figure $E$ shows the distribution of the temperature in relation with the average contact pressure at the same location in the contact area. Each mark represents a complete thread. 


\section{Initial Make-up}

One of the main objective of threaded couplings is to create and maintain a tight seal in order to keep the fluids through the pipes and the environment separated. When no sealing surface is included in the connection design, a thread seal has to be created. An important parameter to create such a seal is the gap size between the thread flanks. This critical gap size is considered to be $150 \mu \mathrm{m}$ [10] when using the API Modified thread compound and should seal the connection for internal pressures up to 275 bar once the thread compound is dried out.

An overview of the results used to determine the minimum and maximum torque position is given in respectively Figure 7 and Figure 8. The value of the maximum torque (2.06 make-up turns) corresponds with the minimal allowed torque defined in literature (2 make-up turns) [2]. This however, does not take into account possible geometric deviations. In addition, the location of the hand tight plane is not unambiguously defined when assembling the connection in the field.

\section{External Loading}

Once the assembly is made up and installed, external forces act on the connection. Two different types of loads are considered within these simulations: axial tension and internal pressure. The first is mainly induced by the weight of the string itself while the latter is caused by the pressure of the fluid within the string. A combination of both loads is usually present in reality. Therefore, the two maxima (indicated as points 2 and 3 in Figure 1) are considered and are calculated as follows [11]:

$$
\begin{gathered}
p_{i, \max }=\frac{2 \sigma}{\sqrt{3}\left(k_{i}+1\right)} \\
\text { at } F_{a}=\frac{\sigma A_{p}\left(k_{i}-1\right)}{\sqrt{3}\left(k_{i}+1\right)} \\
F_{a, \text { max }}=\frac{2 \sigma A_{p} \sqrt{k_{i}^{2}+k_{i}+1}}{\sqrt{3}\left(k_{i}+1\right)} \\
\text { at } p_{i}=\frac{\sigma\left(k_{i}-1\right)}{\sqrt{3} \sqrt{k_{i}^{2}+k_{i}+1}\left(k_{i}+1\right)}
\end{gathered}
$$

With:

$$
\begin{gathered}
k_{i}=\frac{D^{2}+(D-2 t)^{2}}{D^{2}-(D-2 t)^{2}} \\
A_{p}=\frac{\pi}{4}\left[D^{2}-(D-2 t)^{2}\right]
\end{gathered}
$$
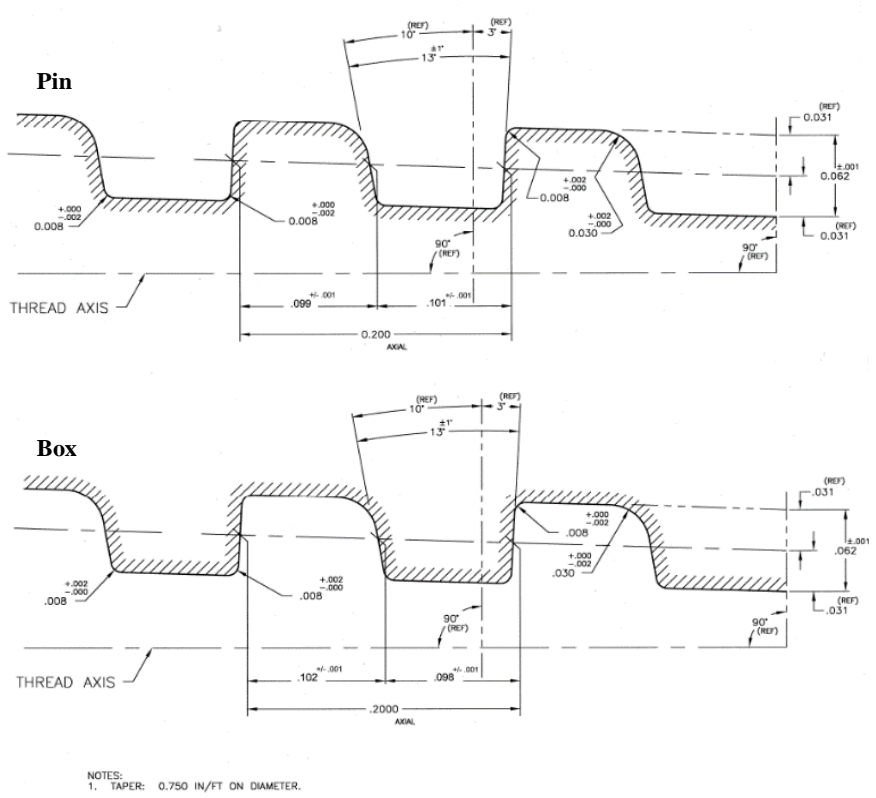

Figure 6: Thread geometry SR23 connection [12]
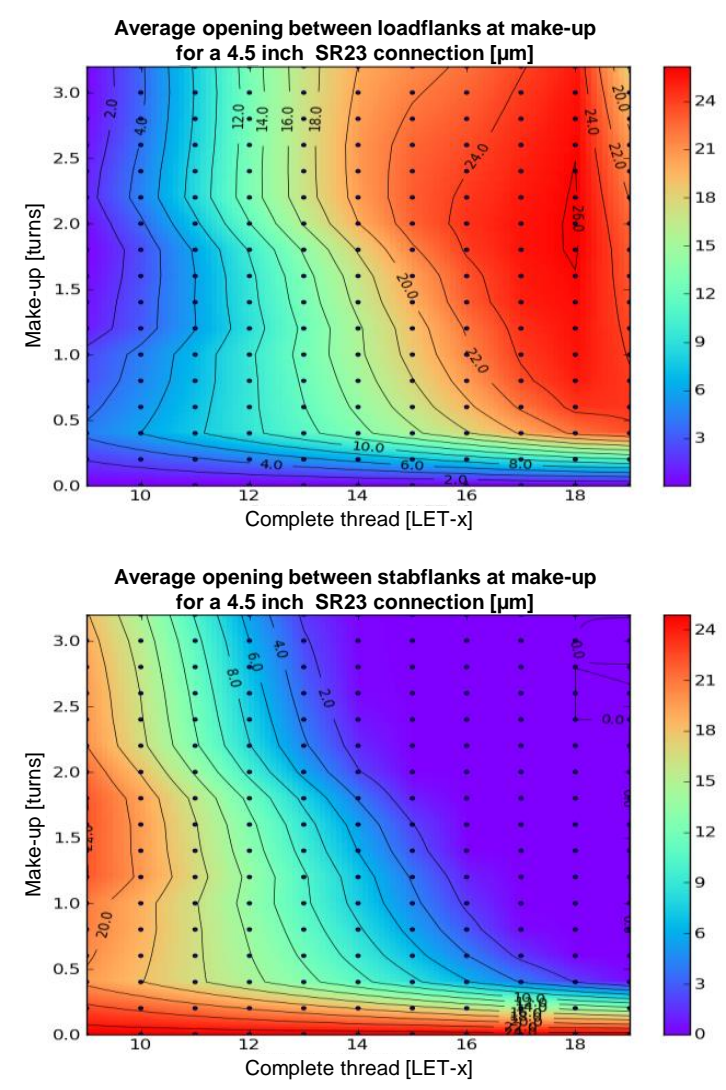

Figure 7: At the complete threads, a crest/root contact is present at the complete threads. Therefore, only the gap size between stab and load flanks needs to be investigated. For the SR23 connection, the critical value is not reached for all make-up positions. The dots represent performed simulations which were used to create the graph using linear interpolation. 


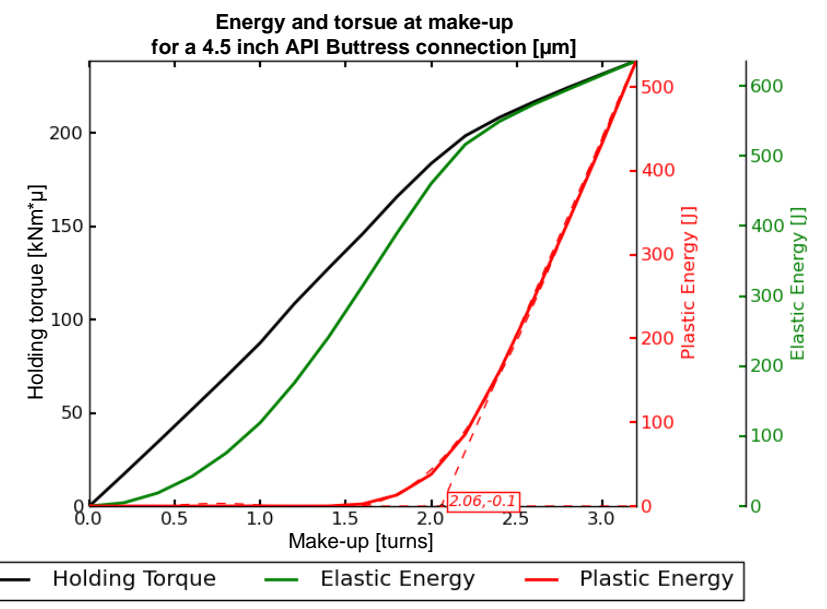

Figure 8: example determine maximum torque

Point 2
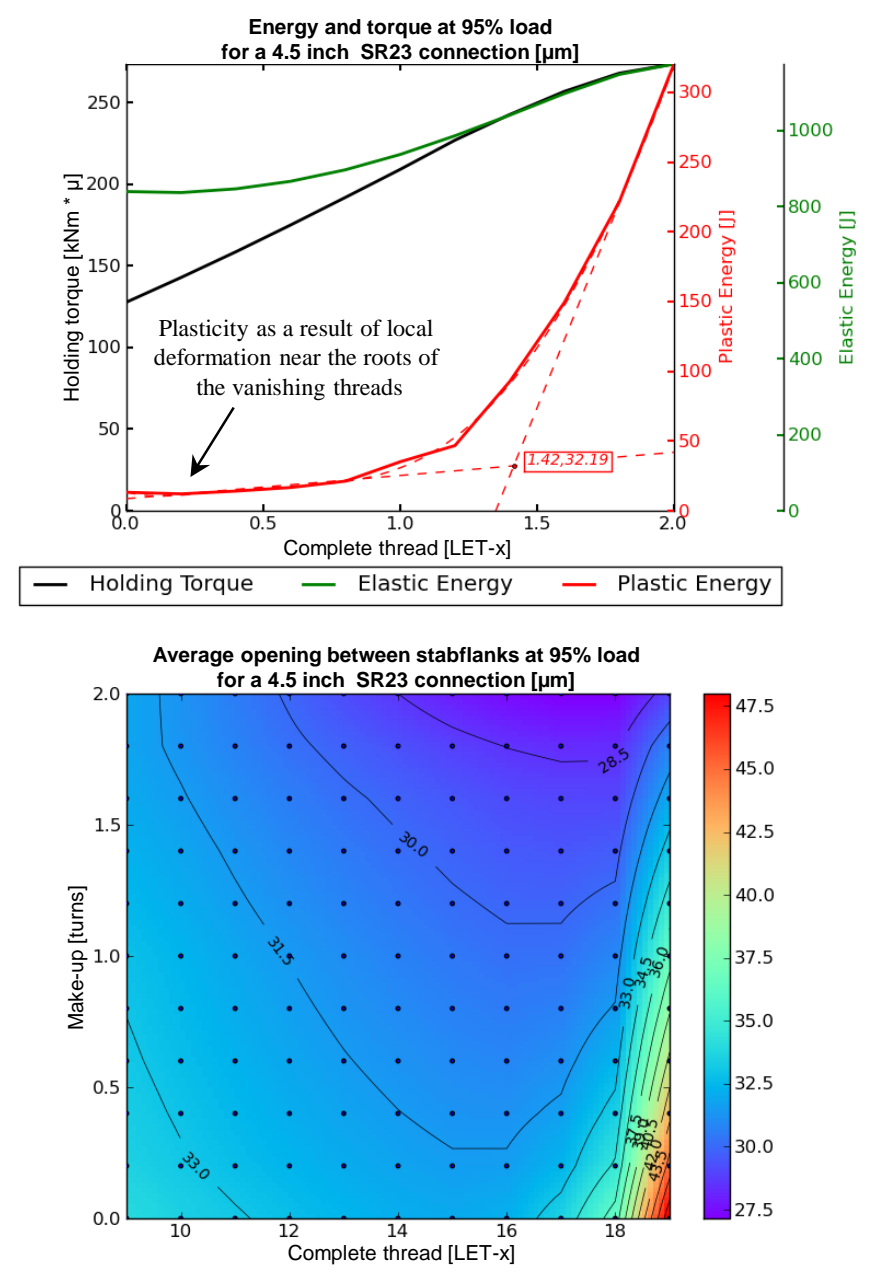

The results used to determine the minimum and maximum make-up position for the different cases when a $100 \%$ load is applied to the SR23 connection are given in Figure 9. It is worth mentioning that not only the absolute gap size, but also the change in gap size relative to the initial make-up values is monitored. This consideration is based on the assumption that after make-up, the dried thread compound clogged the initial gaps and new possible leak paths are created by the additional external loads

\section{DISCUSSION}

When the lower limit for the make-up position needs to be defined, the gap size between the threads at make-up is taken into account. It is visible from Figure 7 that the critical value of $150 \mu \mathrm{m}$ is never reached for both the standard and enhanced buttress connection when using the nominal geometry. However, due to the reduced play present in the SR23 connection, the gap

Point 3
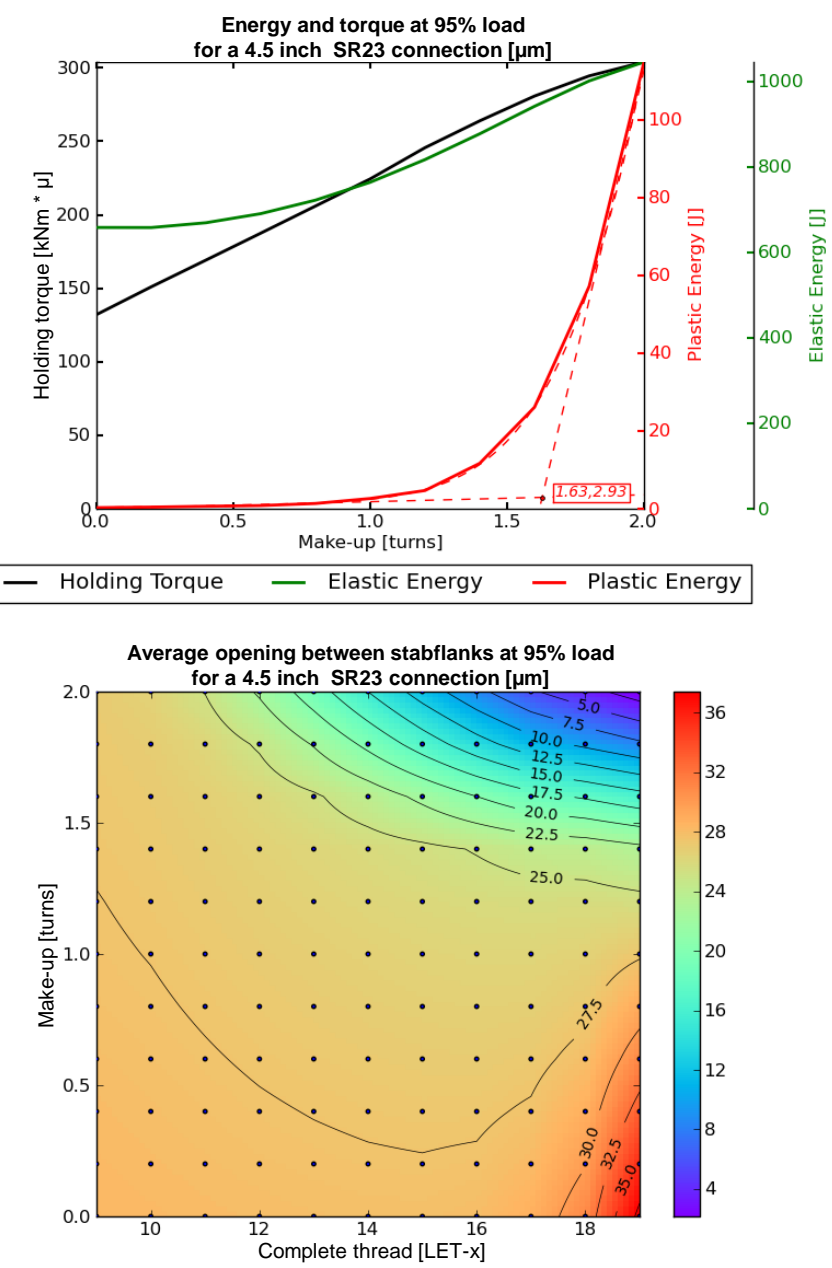

Figure 9: Example of the output used to calculate the minimum and maximum make-up position in case of combined loading. Based on the plastic energy (top), a maximum of 1.4 turns can be applied. The minimum position will depend on the required torque capacity since the maximum gap size appears to be below the critical value in all cases (bottom) 
size is significantly lower. Based on the gap size near all flanks of the vanishing threads, it can be concluded that both connections are supposed to be leak tight up to 275 bar. Nevertheless, due to the reduced space between the stab flanks, higher values can be reached when using the enhanced version, as predicted in literature. Because the minimum position does not appear to be dominated by the play between flanks, the frictional torque has to be considered when attempting to maintain a rigid connection. When the torque required during drilling or installing of the string can be estimated, this value multiplied by a safety factor can be used to determine the minimum required make-up position in which the applied torque can be resisted.

Important to mention is that the relative gap size, given in Figure 10, appears to be similar for both geometries. Knowing that the SR23 has an increased leak resistance [12], it can be concluded that the small relative displacement between pin and box at the complete threads is not indicative for the leak resistance when these types are compared with each other because the crest and root contacts rather than the stab and/or load contacts are dominating during make-up.

In addition to a lower limit, an upper limit for the acceptable make-up position is suggested. The criteria used to determine this limit is the amount of plastic energy in the connection. The ability to reuse connections is often preferred in case the string is broken out (cfr tubing, drill strings) or when a connection has to be broken out and reassembled. When the behavior of the plastic energy for a certain loading condition in function of its make-up (see Figure 8) is considered, two distinct regions can be observed. At low make-up levels, the plastic energy is very limited. An unavoidable and negligible amount of plastic energy can be observed near the vanishing threads as a result of the poisson's ratio in combination with an expanding box and the subtracting pin. When excessive make-up is applied, global deformation after assembly is directly visible at the pin tip and near the tip of the box as illustrated in Figure 11.a. Between both distinct zones, which can be approximated by a straight line, a transition zone is present. The intersection of the two straight lines determine the point which is considered to be the maximum allowable make-up position since the global plastic strains do not exceed the commonly used $0.2 \%$ limit. In addition, the linear relationship between applied torque and rotation starts to reduce as well once this position is exceeded.

When external loads are applied in addition to the initial make-up, an effect on the global plasticity can be observed depending on the load condition. At first, applying tension will cause the connection to deform plastically near the last engaged thread of the pin (see Figure 11.b). When the assembly was initially overtorqued, excessive plastic deformation within this area can be observed before the $100 \%$ load rating is reached. This imposes that the maximum torque obtained by considering only the make-up conditions should be lowered in accordance with the results obtained by the situation in which tension is applied. A second phenomenon which can occur is global plastic deformation in the box (see Figure 11.c). During prior make-up, the pin is shrunk while the box is extended. By applying internal pressure to the assembly, the pin is forced towards or exceeding its initial position while the box is further expanded. When internal pressure is expected, the additional expansion of the box should be taken into account. In order to do this, the maximum allowable make-up position obtained initially should be lowered or steels with a higher yield strength can be used.

After applying this method to determine a minimum and maximum amount of make-up, the results can be compared with values from literature. When buttress connections are used, the minimum and maximum value is determined by a stamped triangle on the pipe. This triangle is located at a distance of 0.400 inch (pipe outer diameter $(\mathrm{OD})=4.5$ "), 0.500 inch $(5 " \leq \mathrm{OD} \leq$
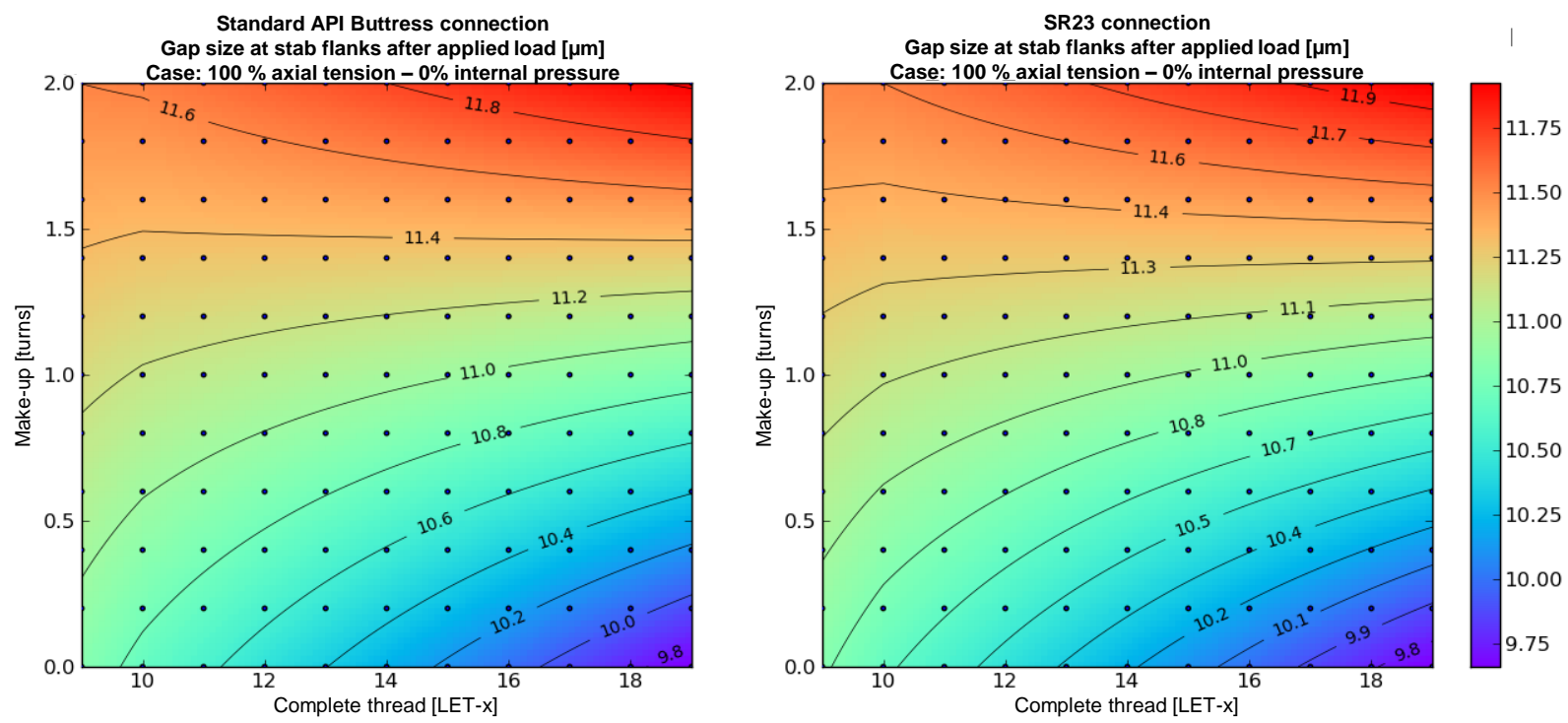

Figure 10: When comparing the increase and/or decrease of the gap size after make-up, both investigated connections show almost identical changes. An example is given for the stab flanks when a $100 \%$ axial tensile load is applied. 
$133 / 8$ ") or 0.375 inch ( $\mathrm{OD} \geq 16$ ") away from the hand tight be increased with 1.875 turns in order to get the maximum allowed number of make-up turns. For the SR23 connections, only a minimum number of 1.1 power turns has to be applied standard BTC connection is used. This difference can be explained by the smaller gap size and higher contact pressures at the flanks. The interchangeability between both connections is assumed to be possible, but the leak tightness cannot be guaranteed in such a case.

\section{CONCLUSIONS}

A numerical study was conducted for a standard API buttress and the enhanced SR23 4.5 inch threaded and coupled connection in an effort to determine the minimum and maximum make-up position, taking into account both axial tension and internal pressure.

In order to determine the minimum position, the gap size between the different thread flanks has to be taken into account to prevent the connection from leaking through the thread helix. In addition, a certain holding torque has to be applied. Using these two parameters, the minimum required amount of make-up turns can be considered the maximum of the number of turns preventing the connection from leaking and the number of turns required to induce a sufficient amount of frictional holding torque.

The criteria used for determining the maximum make-up position is the amount of plastic energy in the connection. When plotting the amount of plastic energy in function of applied load and make-up, a distinction between local (which is unavoidable at the incomplete threads) and global plastic deformation can be made. The maximum number of make-up turns is considered to be the intersection of the linear trends of both the local and plastic deformation.

In addition to the numerical study, an experimental setup was designed for the validation of the used model during the make-up stage using different techniques. The strains were validated using a combined approach using digital image correlation and strain gauges. When taking into account the possible taper mismatch, a good correspondence with the numerical model could be found. In addition, a promising attempt was made to validate the distribution of the contact pressures by measuring the surface temperature of the box. Because the area of the largest deformations did not correspond with the location of maximum temperature, the deformation energy could be ruled out as the major heat source. Focusing on the frictional energy, the location of highest temperature appeared to correspond with the location of the highest contact pressures at the threads.

\section{REFERENCES}

[1] Galle, T., De Waele, W., Van Wittenberghe, J., and De Baets, P., 2014, "Comparison of 2D and 3D Finite Element Models to Predict the Make-up Torque in Threaded Connections", Journal of Pressure Vessel Technology, submitted.

[2] American Petroleum Institute, 2005, "API 5CT: Specifications for Casing and Tubing"
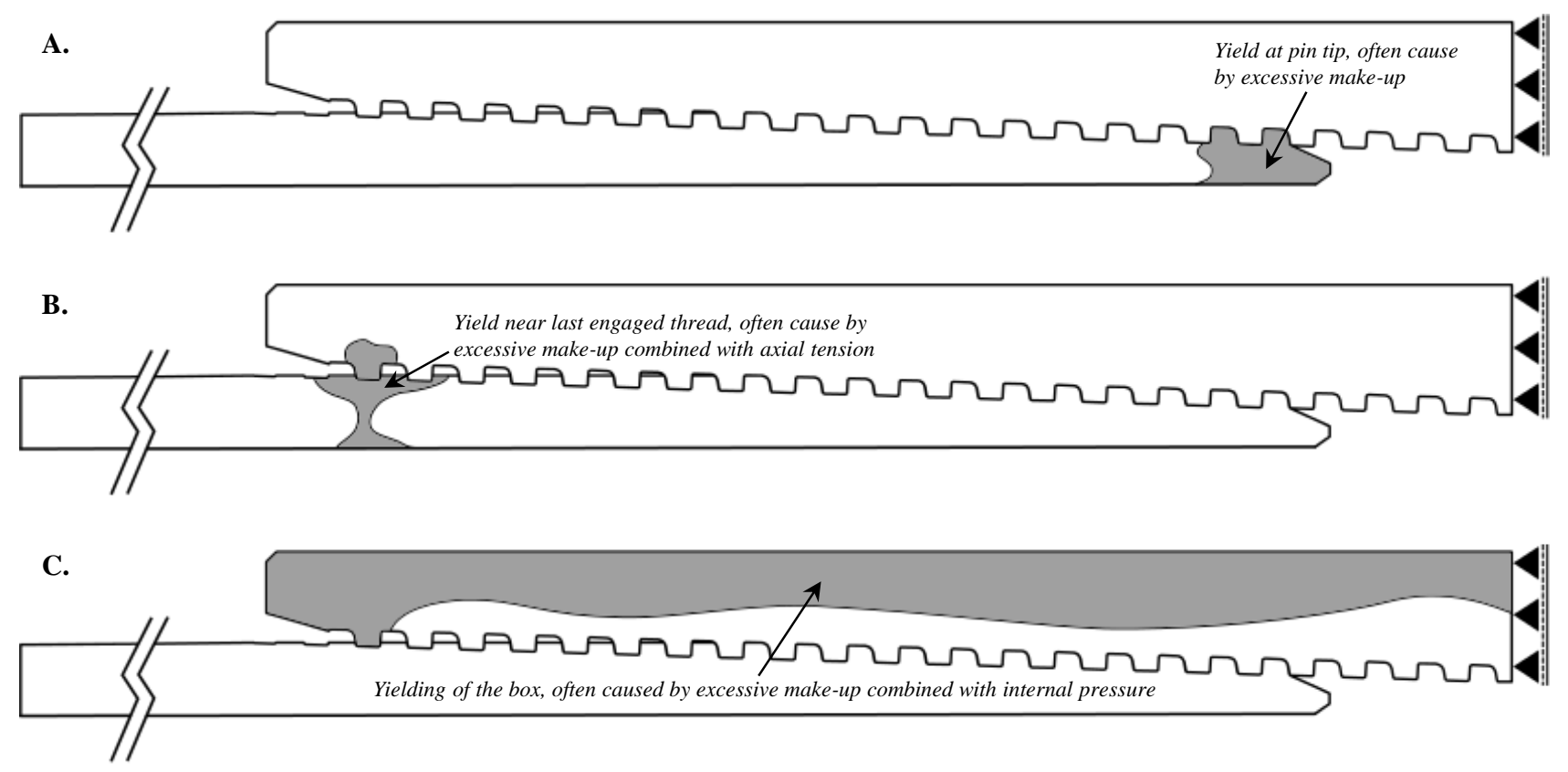

Figure 11: Schematic overview of the location where plastic zones often appears when no adequate make-up is applied (A: within the pin tip, B: near the last engaged thread, C: overall yielding of the box). 
[3] Galle, T., De Waele, W., and De Baets, P., 2013, "Effect of Make-up on the Structural Performance of Standard Buttress Connections Subjected to Tensile Loading”, Proceedings of the 2013 Pressure Vessels \& Piping Division Conference, PVP 2013-97282

[4] Van Wittenberghe, J., Galle, T., De Baets, P., and De Waele, W., 2011, "Numerical modeling and experimental validation of a threaded pipe connection under axial tension", Mechanical Engineering Letters, 5, pp. 89-94

[5] Kawashima, H., 1990, "Effect of Incomplete Threads on the Jump-Out Tensile Failure of Premium Connections for Oil or Gas Wells", JSME International Journal, 33 (1), pp. 107-112

[6] Hertelé, S., Verstraete, M., Van Minnebruggen, K., Denys, R., and De Waele, W., 2013, "Applications of Digital Image Correlation in Girth Weld Testing", Pipeline Technology Conference, Ostend, Belgium.

[7] Galle, T., De Pauw, J., De Waele, W., Van Wittenberghe, J., and De Baets, P., 2014, "Validating Numerically Predicted Make-up of Threaded Connections using Digital Image Correlation and Infrared Monitoring”, Journal of Strain Analysis for Engineering Design, submitted.
[8] Parkus H., 1976, “Thermoelasticity”, Springer Vienna.

[9] American Petroleum Institute, 1996, “API 5B: Specification for Threading, Guaging, and Thread Inspection of Casing, Tubing and Line Pipe Threads"

[10] Watts, J. D., and Ramos, B. W., 2004, "Wedgethread Pipe Connection", US Patent 6,682,101 B2, Austin, Texas

[11] European Committee for Standardization, 2006, "ISO 13679: Petroleum and Natural Gas Industries - Procedures for Testing Casing and Tubing Connections"

[12] American Petroleum Institute, 2010, "SR 23 Revisions to API St. 5B Appendix E"

[13] American Petroleum Institute, 2009, "Attachment 10 SRXX-BTC 5C1 DEC2009"

[14] American Petroleum Institute, 1996, “API 5B: Specifications for Threading, Gauging, and Thread Inspection of Casing, Tubing, and Line Pipe Threads"

[15] American Petroleum Institute, 2009, “API SR23 5CT Appendix B" 